\title{
HYDROPHOBIC-LIKE SOLVATION IN NON-AQUEOUS SOLUTIONS: POSITRON ANNIHILATION IN AND COMPRESSIBILITY OF TERT-BUTANOL AND TETRAMETHYLUREA IN ETHYLENE GLYCOL
}

\author{
K. Jerie ${ }^{a}$, A. Baranowski ${ }^{a}$, J. GLiński $^{b}$ And J. PrZybylski ${ }^{b}$ \\ ${ }^{a}$ Institute of Experimental Physics, University of Wrocław \\ Pl. Maxa Borna 9, 50-204 Wrocław, Poland \\ ${ }^{b}$ Faculty of Chemistry, University of Wrocław \\ F. Joliot-Curie 14, 50-383 Wrocław, Poland
}

\begin{abstract}
The pioneer experimental results of positron annihilation experiments in non-aqueous solutions of tetramethylurea and tert-butanol were compared to those of ultrasonic velocity. For tert-butanol, the positron annihilation results exclude existence of hydrophobic-like interactions and formation of weak, labile crystalline-like solvates in solution. For tetramethylurea solute the changes of annihilation parameters with concentration are minor and only limited conclusions can be drawn on this system, while they are stronger for tert-butanol solute. However, all the above is consistent with an assumption that the more hydrophobic solute (tert-butanol) should affect the original glycol structure stronger than that interacting with glycol via hydrogen bondings only. On the other hand, the ultrasonic data for the systems tested, although very precise, hardly confirm formation of any specific structure. It is possible, however, that the solvates do not differ in their compressibility from the pure liquids, and/or are formed only in very small amounts. Thus, usefulness of the positron annihilation method in investigation of liquid structure was proven.
\end{abstract}

PACS numbers: 61.25.Em, 78.70.Bj

\section{Introduction}

So far we investigated, using two independent experimental methods, positron annihilation and ultrasonic velocity, the structure of a series of systems where a non-electrolyte was dissolved in water. They were mainly solutes with hydrophobic parts [1-8], and their interactions with water framework could be explained 
in terms of hydrophobic hydration $[9,10]$. The systems with hydrophilic solutes were tested, too [11-16]. The positron annihilation method has been found to be a valuable tool in these investigations, especially when completed by the ultrasonic one.

Formation, as well as lifetime of ortho-positronium, which is responsible for the long-lived mechanism (in this paper indexed as three), is strongly influenced by the physical properties of the medium surrounding the positronium pseudoatom. The liquid water structure, with cavities and open hydrogen-bonded network, can be modified and then stabilised by the presence of a guest molecule. Non-electrolytes with hydrophobic parts form strong hydrates of quasi-clathrate architecture, where considerably big voids are occupied by solute molecules. Thus, the liquid structure should become more open-work, but also more rigid. The competition of these two effects yields characteristic concentration dependences of $\tau_{3}$ and $I_{3}$ : the former exhibits an inflection and the latter one - a maximum at the concentration corresponding to the stoichiometry of the hydrates formed; see our previous papers cited in this paper.

One can extract the same stoichiometry from ultrasound velocity data. From Laplace's equation $\beta=\left(\rho c^{2}\right)^{-1}$ the adiabatic compressibility coefficient $\beta$ can be calculated ( $\rho$ - density of liquid, $c$ - sound velocity). When plotting the adiabatic compressibilities $\beta$ against non-electrolyte concentration in water, the isotherms of $\beta$ intersect usually at a common point. The $X$-coordinate of this point corresponds to the stoichiometry of quasi-clathrate hydrates, according to the concept of Endo [17]. The details of this interpretation are given in [18, 19]. It is worth noting that the compressibility value at the crossing point should be and is, in most cases, very close to that found by von Stackelberg for solid clathrates [20].

Most of our previous papers concerned the systems of aqueous solutions of non-electrolytes, or tetraalkylammonium salts, where the solute molecule has a well-defined hydrophobic part, which allows the hydrophobic hydration.

While the hydrophobic hydration means, in general, the formation of a specific architecture of hydrogen-bonded water molecules around the hydrophobic part of the solute (or the solute molecule as a whole, like for hydrocarbons) and needs destruction of the original solvent structure, the question arises if such phenomenon can be found uniquely in aqueous solutions. It seems obvious that the solvent should have a strong, three-dimensional network of hydrogen bondings, similar to water.

A very good candidate is ethylene glycol, $\mathrm{HO}-\mathrm{CH}_{2}-\mathrm{CH}_{2}-\mathrm{OH}$. The high viscosity $\left(19.9 \mathrm{cp}\right.$ at $\left.20^{\circ} \mathrm{C}\right)$ and values of melting $\left(-11.5^{\circ} \mathrm{C}\right)$ and boiling $\left(198^{\circ} \mathrm{C}\right)$ temperatures [21] suggest its possible structural similarity to water in liquid phase. Thus, it seemed interesting to apply the same experimental procedure to compare aqueous and ethylene glycol solutions of substances with less or more distinct hydrophobic groups.

Two solutes chosen for comparison differ significantly in their interactions with the surrounding medium. Tert-butyl alcohol, $\mathrm{CH}_{3}-\mathrm{CH}\left(\mathrm{CH}_{3}\right)(\mathrm{OH})-\mathrm{CH}_{3}$, is a typical hydrophobic solute, while hydrophobicity of tetramethylurea, $\left(\mathrm{CH}_{3}\right)_{2} \mathrm{~N}-\mathrm{CO}-\mathrm{N}\left(\mathrm{CH}_{3}\right)_{2}$, is limited and its interactions with polar solvent are much more complex. 


\section{Experimental}

\subsection{Chemicals}

Ethylene glycol (>99\%, from Baker), tert-butanol (99.3\%, from Aldrich) and tetramethylurea (for synthesis, from Merck) were glass-distilled under reduced pressure. Solutions were prepared by weighing.

\subsection{Positron annihilation measurements}

The annihilation measurements were carried out at room temperature (approx. $25^{\circ} \mathrm{C}$ ) with a standard measuring device based on the "fast-slow" coincidence technique as described elsewhere [1]. The obtained spectra were resolved using POSITRONFIT program.

\subsection{Sound velocity measurements}

The ultrasonic velocity was measured with an accuracy of $\pm 0.1 \mathrm{~m} / \mathrm{s}$ using a "sing-around" equipment (apparatus Ecolab MPFU, Poland). The details of the apparatus and measurement techniques applied in this study are given in [22]. For solutions strongly absorbing the sound wave an apparatus UI 12, originally constructed in the Institute for Fundamental Technical Problems, Polish Academy of Sciences, Warsaw, was used (interferometric accuracy of $0.4 \mathrm{~m} / \mathrm{s}$ ).

\subsection{Density measurements}

Density was measured using a vibrating pipe method (apparatus Ecolab MG-2, Poland). The accuracy was not worse than $\pm 0.02 \mathrm{~kg} / \mathrm{m}^{3}$.

\section{Experimental results}

Both the solutes, tert-butanol and tetramethylurea, are fully soluble in ethylene glycol in the temperature range tested.

\subsection{Sound velocity}

The adiabatic compressibility coefficients $\beta$ were calculated from Laplace's equation $\beta=\left(\rho c^{2}\right)^{-1}$, where $\rho$ - density of the liquid, $c$ - sound velocity. The concentration dependences of the compressibility are shown in Fig. 1.

Of course, no intersection of isotherms of adiabatic compressibility could be observed in the systems under test, while both the components have the same sign of the temperature derivative of compressibility.

The lack of characteristic points (extrema, inflections) in the derivative $\mathrm{d} \beta / \mathrm{d} T$ suggests that no specific solvate structures are formed when adding tert-butanol or tetramethylurea to ethylene glycol. 


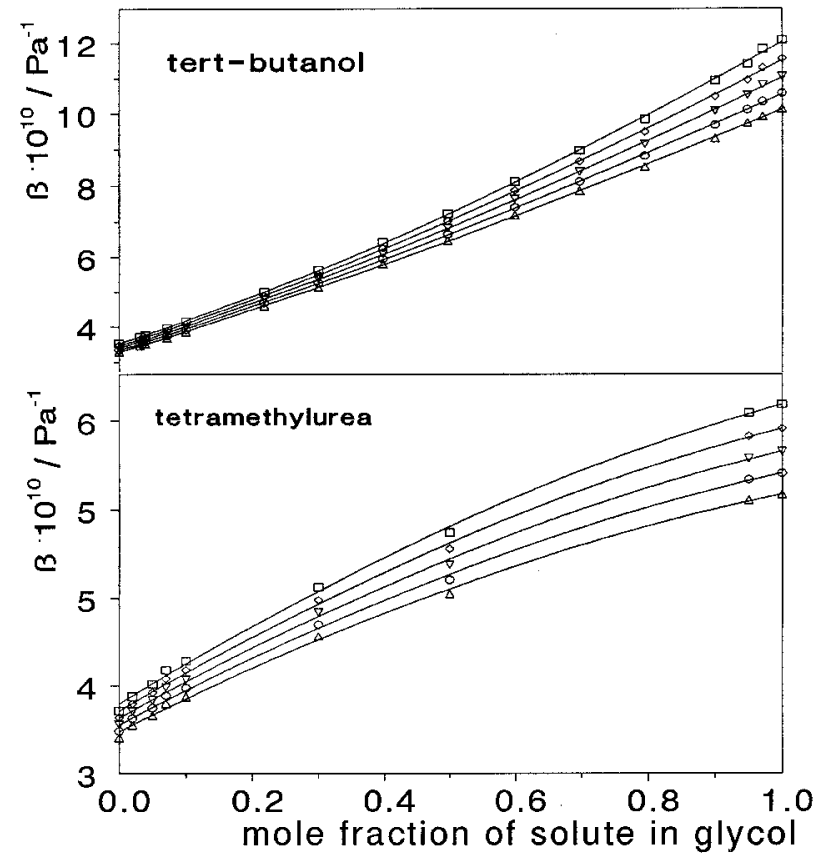

Fig. 1. Plots of adiabatic compressibility coefficients vs. mole fraction of tert-butanol (upper graph) and tetramethylurea (lower graph) in ethylene glycol. $\nabla-298 \mathrm{~K}, \mathrm{O}$ $303 \mathrm{~K}, \nabla-308 \mathrm{~K}, \diamond-313 \mathrm{~K}, \square-318 \mathrm{~K}$. Lines are drawn arbitrarily (spline function).

\subsection{Positron mean lifetimes}

The annihilation spectra were resolved into three components with lifetimes $\tau_{1}, \tau_{2}, \tau_{3}$ and corresponding intensities $I_{1}, I_{2}, I_{3}$.

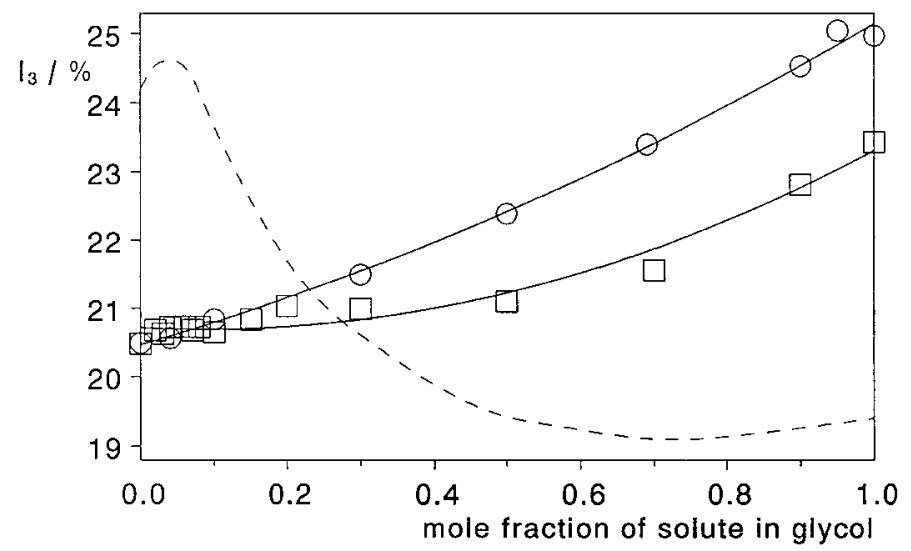

Fig. 2. Intensities $I_{3}$ of the long-lived component of annihilation spectra vs. solute concentration in ethylene glycol. Circles - tert-butanol, squares - tetramethylurea, broken line - the results for aqueous solutions of ethanol [6]. 


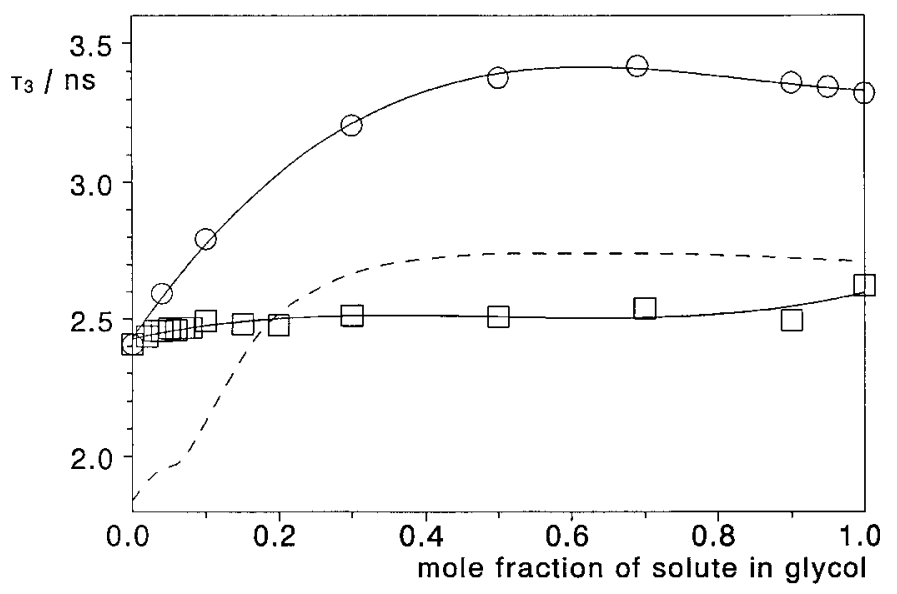

Fig. 3. Lifetimes $\tau_{3}$ of the long-lived component of annihilation spectra vs. solute concentration in ethylene glycol. Circles - tert-butanol, squares - tetramethylurea, broken line - the results for aqueous solutions of ethanol [6].

The concentration dependences of the long-lived component's parameters $\left(\tau_{3}\right.$ and $\left.I_{3}\right)$ are shown in Figs. 2 and 3. For comparison, the corresponding literature results obtained for the system water + ethanol [6] (a system with dominating hydrophobic hydration, where quasicrystalline clathrate-like hydrates are formed) are also shown in these figures.

Changes of both $\tau_{3}$ and $I_{3}$ with solute concentration are weak and do not resemble those observed in water+ethanol. The shape of $\tau_{3}$ suggests, however, non-ideality of the system glycol+tert-butanol and seems worth of a deeper analysis.

\section{Discussion}

The formation of clathrate hydrates in the aqueous solutions of non-electrolytes with hydrophobic parts of their molecule is well proved now. According to the concept of the iceberg formation of Frank and Evans [1] or to that of hydrophobic hydration formulated by Franks [2], when an organic compound is dissolved in water, the water structure is modified around the non-polar parts of the solute molecule in the direction of greater "crystallinity", whatever the actual quasi-solid water structure induced in this way. In the vicinity of guest molecule the water structure is determined mainly by its mobility. Molecules of solute are accommodated in the cavities of the open hydrogen-bonded network, which in pure water are most probably occupied by "monomeric" water molecules [23-26].

Judging from the experimental data shown above, none of these phenomena occur in the systems under investigation. Our assumption on specific, glycol-rich structures was initially supported by the paper of Chaban et al. [27], where the light scattering intensity in ethylene glycol + tetramethylurea was reported and its sharp maximum around TMU mole fraction equal to 0.05 was found. (This is 
why we performed more measurements in the region of low TMU concentrations compared to tert-butanol solute.) In the light of our experiments, however, no strong structural process occurs in this system. Also close-critical phenomena are not probable in such the low TMU concentrations. All the above make the findings of Chaban et al. rather doubtful.

There is, however, a weak structural process possible in the system glycol+tert-butanol. According to the concept of Levay, Vertes, and Hautojarvi, for an ideal mixture of classical liquids the long-living lifetime should be a simple function of concentration [28]

$$
\tau_{3, \mathrm{AB}}^{-1 / 2}=\left[\tau_{3, \mathrm{~A}}^{-1 / 2} V_{\mathrm{A}}-\left(\tau_{3, \mathrm{~A}}^{-1 / 2} \mathrm{~V}_{\mathrm{A}}-\tau_{3, \mathrm{~B}}^{-1 / 2} \mathrm{~V}_{\mathrm{B}}\right) \mathrm{X}_{\mathrm{B}}\right] \frac{1}{V_{\mathrm{AB}}}
$$

where subscripts $\mathrm{A}, \mathrm{B}$, and $\mathrm{AB}$ denote pure components $\mathrm{A}$ and $\mathrm{B}$, and their mixture, respectively; $V$ is the molar volume. In the case of a mixture its mean molar volume $V_{\mathrm{AB}}$ can be calculated from the molar volumes of the components: $V_{\mathrm{AB}}=\left(X_{\mathrm{A}} M_{\mathrm{A}}+X_{\mathrm{B}} M_{\mathrm{B}}\right) / \rho(M-$ molecular mass $)$. Figure 4 illustrates the differences between the experimental lifetimes of the long-living component of the annihilation spectrum and those calculated using the above formula.

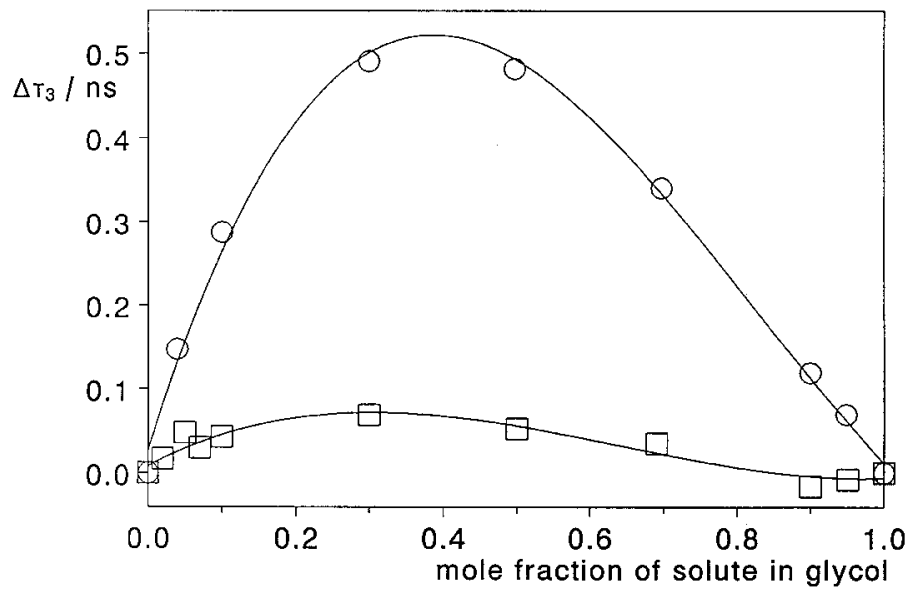

Fig. 4. "Excess" lifetimes $\tau_{3}$ of the long-lived component of annihilation spectra vs. solute concentration in ethylene glycol, calculated as differences of the experimental ones and those from equation of Levay, Vertes, and Hautojarvi. Circles - tert-butanol, squares - tetramethylurea.

The deviations from "ideality" in Fig. 4 are much greater for tert-butanol than for tetramethylurea. Note, however, that for the latter the maximum deviation occurs at a solute mole fraction close to 0.3 - the number suggesting the formation of 2:1 solvates, while for tert-butanol it is shifted towards higher alcohol concentrations, and the postulated structure of its glycol solution could be a mixture of solvates with stoichiometries $1: 1$ and $2: 1$. Nevertheless, such compositions exclude any clathrate-like structure, which should be much more solvent-rich.

According to the previous discussion, it is surprising that the ultrasonic data for the systems tested, although very precise, hardly confirm formation of 
any specific structure or solvates. It is possible, however, that the solvates almost do not differ in their compressibility from the pure liquids, and/or are formed only in very small amounts.

Thus, our initial assumption on structural similarity of aqueous and ethylene glycol solutions of non-electrolytes found no confirmation. Both the experimental methods applied are univocal and show that in the solutions under investigation only very weak solvates are formed. It seems interesting that complexity and the number of hydrogen bondings formed by a solvent is not a sufficient condition for strong solvent-solute interactions and formation of crystalline-like hydrates.

Concluding, the aim of this work which was to check if ethylene glycol is able, like it is in water, to arrange its molecules around the hydrophobic fragment, forming cages of crystalline-like architecture, was not confirmed. This result suggests that ethylene glycol does not form, like water, an open-work structure in liquid state, and no hydrophobic-like hydration is possible in this solvent. It remains open to question, however, if other 1,2-glycols behave like ethylene one. This will be the subject of our future investigations.

\section{Acknowledgments}

This work was partially supported by the Committee for Scientific Research, grant No. 2016/GW/IFD/2000.

\section{References}

[1] K. Jerie, A. Baranowski, B. Rozenfeld, S. Ernst, J. Gliński, Acta Phys. Pol. A 64, $77(1983)$

[2] K. Jerie, A. Baranowski, B. Rozenfeld, S. Ernst, B. Jeżowska-Trzebiatowska, J. Gliński, Acta Phys. Pol. A 66, 3 (1984).

[3] K. Jerie, A. Baranowski, B. Rozenfeld, S. Ernst, B. Jeżowska-Trzebiatowska, J. Gliński, Acta Phys. Pol. A 66, 167 (1984).

[4] K. Jerie, A. Baranowski, S. Ernst, J. Gliński, Acta Phys. Pol. A 69, 81 (1986).

[5] K. Jerie, A. Baranowski, S. Ernst, J. Gliński, Acta Phys. Pol. A 69, 91 (1986).

[6] K. Jerie, A. Baranowski, B. Rozenfeld, J. Gliński, S. Ernst, Acta Phys. Pol. A 73, 667 (1988).

[7] K. Jerie, A. Baranowski, B. Rozenfeld, B. Jeżowska-Trzebiatowska, J. Gliński, Acta Phys. Pol. A 79, 507 (1991).

[8] K. Jerie, A. Baranowski, B. Rozenfeld, B. Jeżowska-Trzebiatowska, J. Gliński, Acta Phys. Pol. A 82, 471 (1992).

[9] H.S. Frank, M.W. Evans, J. Phys. Chem. 13, 507 (1945).

[10] F. Franks, Ann. N.Y. Acad. Sci. 125, 277 (1965).

[11] A. Baranowski, K. Jerie, J. Gliński, K. Orzechowski, Acta Phys. Pol. A 88, 59 (1995).

[12] K. Jerie, A. Baranowski, J. Gliński, in: Proc. of the 31st Seminar on Positron Annihilation, Jarnołtówek (Poland), Sept. 27-Oct. 1 1999, Eds. W. Świątkowski, K. Jerie, Opole University, Opole 2000, p. 103. 
[13] K. Jerie, A. Baranowski, J. Gliński, J. Przybylski, J. Radioanal. Nuclear Chem. 246, 343 (2000).

[14] K. Jerie, A. Baranowski, J. Gliński, K. Orzechowski, Acta Phys. Pol. A 85, 563 (1994).

[15] Gy. Jákli, A. Baranowski, K. Jerie, J. Gliński, K. Orzechowski, Bull. Pol. Acad. Sci. - Chem. 42, 67 (1994).

[16] Gy. Jákli, A. Baranowski, K. Jerie, J. Gliński, K. Orzechowski, Nukleonika 42, 123 (1997) (tetrabutylammonium bromide + water); Gy. Jákli, K. Jerie, A. Baranowski, J. Gliński, Acta Phys. Polon. A 93, 649 (1998) (tetramethylammonium chloride + water). Note, however, that for tetraalkylammonium salts the hydrophobic interactions compete with the electrostatic ones, making interpretation of both positron annihilation and ultrasonic results much more difficult than for non-electrolytes.

[17] H. Endo, Bull. Chem. Soc. Jpn. 16, 1586 (1973).

[18] S. Ernst, J. Gliński, Pol. J. Chem. 56, 339 (1982).

[19] S. Ernst, J. Gliński, Mater. Sci. 3, 68 (1977).

[20] M. von Stackelberg, H. Müller, Z. Elektrochem. 58, 25 (1954).

[21] CRC Handbook of Chemistry and Physics, Ed. R.C. Weast, 56th ed., CRC Press, Cleveland (Ohio, USA) 1975.

[22] S. Ernst, J. Gliński, B. Jeżowska-Trzebiatowska, Acta Phys. Polon. A 64, 501 (1979).

[23] L. Pauling, The Nature of the Chemical Bond, 3rd ed., Cornell University Press, Ithaca - New York 1960, p. 469.

[24] H.S. Frank, A.S. Quist, J. Chem. Phys. 34, 604 (1961).

[25] V.N. Yashkitshev, O.Ya. Samoilov, Zh. Strukt. Khim. 3, 211 (1962).

[26] M.N. Buslayeva, O.Ya. Samoilov, Zh. Strukt. Khim. 4, 502 (1963).

[27] I.A. Chaban, M.N. Rodnikova, J. Barthel, L.L. Chaikov, S.V. Krivokhiza, V.V. Zhackova, J. Mol. Liq. 80, 27 (1999).

[28] B. Levay, A. Vertes, P. Hautojarvi, J. Phys. hem. 77, 2229 (1973). 